\title{
IdeAs
}

Idées d'Amériques

$16 \mid 2020$

Les marges créatrices : intellectuel.le.s afrodescendant.e.s et indigènes auX Amériques, XIX-XXe siècle

\section{Quand les femmes des favelas prennent la plume : la force de l'écriture comme témoignage chez Carolina Maria de Jesus (1914-1977) et Conceição Evaristo (1946-...)}

When favela women take up their pens: testimony in the works of Carolina Maria de Jesus (1914-1977) and Conceição Evaristo (1946-...)

Quando as mulheres da favela se tornam escritoras: a força da escrita como testemunho em Carolina Maria de Jesus (1914-1977) e Conceição Evaristo (1946...)

\section{Silvia Capanema}

\section{OpenEdition Journals}

Édition électronique

URL : http://journals.openedition.org/ideas/9552

DOI : 10.4000/ideas.9552

ISSN : 1950-5701

Éditeur

Institut des Amériques

Référence électronique

Silvia Capanema, «Quand les femmes des favelas prennent la plume : la force de l'écriture comme témoignage chez Carolina Maria de Jesus (1914-1977) et Conceição Evaristo (1946-...) », IdeAs [En ligne], 16 | 2020, mis en ligne le 13 octobre 2020, consulté le 18 octobre 2020. URL : http:// journals.openedition.org/ideas/9552; DOI : https://doi.org/10.4000/ideas.9552

Ce document a été généré automatiquement le 18 octobre 2020

IdeAs - Idées d'Amériques est mis à disposition selon les termes de la licence Creative Commons Attribution - Pas d'Utilisation Commerciale - Pas de Modification 4.0 International. 


\section{Quand les femmes des favelas prennent la plume : la force de l'écriture comme témoignage chez Carolina Maria de Jesus (1914-1977) et Conceição Evaristo (1946-...)}

When favela women take up their pens: testimony in the works of Carolina Maria de Jesus (1914-1977) and Conceição Evaristo (1946-...) Quando as mulheres da favela se tornam escritoras: a força da escrita como testemunho em Carolina Maria de Jesus (1914-1977) e Conceição Evaristo (1946...)

\section{Silvia Capanema}

\section{Introduction}

1 La faim a une couleur, " elle est jaune ${ }^{1}$ ». Avec ces mots, Carolina Maria de Jesus a provoqué l'étonnement et la fascination à l'époque de la publication de son journal au Brésil, Quarto de Despejo (1960), traduit en France comme Le Dépotoir ${ }^{2}$. Le récit, avec son sous-titre, "journal d'une habitante de la favela", impressionne par sa richesse en détails et parce qu'il porte sur une réalité méconnue et pourtant si proche de la «bonne société brésilienne » qui lisait la presse et les revues illustrées dans les années 1950, où quelques extraits du journal sont publiés pour la première fois (Dans A Folha da Noite, en 1958, et dans O Cruzeiro, en 1959) 3 . On apprend que l'auteure de ces textes était une femme noire âgée de quarante-quatre ans, qui était "catadora de papel », (chiffonnière ou « ramasseuse de papier ») dans le Brésil des années 1950.

Carolina Maria de Jesus est née à Sacramento, une ville de province dans l'État du Minas Gerais, en 1914 et est décédée en 1977 à São Paulo4. Elle était petite fille 
d'esclaves et une des sept enfants d'une mère blanchisseuse. Dans les années 1930, sa famille migre pour la région de Franca, dans l'intérieur de l'État de São Paulo, participant à un mouvement de migrations intérieures au Brésil (de villes appartenant aux États plus périphériques vers les grandes villes, notamment São Paulo et Rio de Janeiro).

3 Après le décès de sa mère, Maria Carolina de Jesus part vivre à la métropole de São Paulo, à l'âge de vingt-six ans, où elle devient tout d'abord employée domestique. En 1948, elle s'installe dans la favela du Canindé, où elle donne naissance à trois enfants, deux garçons et une fille, tous les trois de pères différents. Caroline les élève seule.

Carolina Maria de Jesus devient aussi « ramasseuse de papier» (catadora de papel), un métier proche de celui des chiffonniers en France. Elle aimait écrire son journal, des poèmes, des pièces, composait des chansons, entre autres. Son journal a été découvert par le journaliste Audálio Dantas en 1958. Il devient par la suite un best-seller et une référence.

5 Avec le succès du livre, publié dans treize langues, Carolina sort de la favela et va vivre dans une maison dans la banlieue de São Paulo. Elle continue d'écrire, de composer, ce qu'elle fera toute sa vie, et publie encore quatre livres dont un édité en France à l'origine et publié comme œuvre posthume au Brésil ${ }^{5}$. Malgré le succès de ses premiers livres et l'intérêt qu'ils suscitent par leur aspect "exotique » auprès des lecteurs du monde entier, Carolina Maria de Jesus termine sa vie dans des conditions très précaires, et presque oubliée.

6 Mais l'écriture de Carolina Maria de Jesus n'a pas seulement touché les lectrices et les lecteurs des classes aisées au Brésil et à l'étranger. Elle a aussi été une source d'inspiration et de dialogue pour d'autres femmes pauvres afro-descendantes et qui vivaient dans différentes favelas au Brésil. C'est le cas de Conceição Evaristo, née en 1946 au Minas Gerais, le même État que Carolina Maria de Jesus, qui était aussi une écrivaine noire d'origine populaire, une référence majeure aujourd'hui de la littérature contemporaine brésilienne. Dans l'avant-propos de l'édition française de son livre L'histoire de Poncia, Conceição Evaristo revient sur l'importance du travail de Carolina Maria de Jesus et ce que sa pensée a apporté pour l'histoire de sa famille:

Quand Quarto de despejo [Le Dépotoir], le journal de Maria Carolina de Jesus, fut publié en 1960, causant un vif émoi chez les lecteurs des classes favorisées brésiliennes, nous nous identifiâmes immédiatement à l'auteure. Comme Carolina Maria de Jesus dans les rues de São Paulo, nous connaissions dans celles de Belo Horizonte l'odeur et la saveur des poubelles, mais aussi le plaisir que les restes des riches pouvaient procurer. Manquant des choses essentielles au quotidien, les excédents des uns presque toujours construits sur la misère des autres - revenaient humblement entre nos mains. Les restes.

Ma mère lut le journal de Carolina Maria de Jesus et décida, quelques années plus tard, d'écrire le sien. Je conserve ces écrits avec moi afin de prouver un jour que Carolina Maria de Jesus, favelada de Canindé, quartier nord de São Paulo, a créé une tradition littéraire. Une autre favelada de Belo Horizonte a suivi le chemin de l'écriture initiée par Carolina et a écrit, elle aussi sous la forme d'un journal, la misère du quotidien qu'elle affrontait.

(Conceição, E., 2015)

Dans ce passage, Conceição Evaristo se reconnaît dans l'histoire de Carolina Maria de Jesus, à travers l'expérience de la misère et le fait d'être exclue dans une grande ville brésilienne. Mais aussi, cet extrait révèle que le désir d'écriture, d'une certaine manière, aurait vu le jour chez Conceição Evaristo à travers sa mère, inspirée par 
l'exemple de Carolina Maria de Jesus. Non seulement le désir d'écrire, mais aussi le fait de croire qu'écrire était possible.Conceição Evaristo est née en 1946 à Belo Horizonte ${ }^{6}$. Femme noire d'origine très modeste, elle a grandi dans la favela Pindura Saia dans la région Sud de la ville, un quartier qui fait partie du projet de desfavelização (le démantèlement programmé de la favela, suivi d'un processus radical de gentrification $\mathrm{du}$ quartier), expulsant les populations locales pour le transformer en un quartier bourgeois. Elle a pu aller à l'école publique, était très bonne élève et dès son plus jeune âge fut très intéressée par la lecture et l'écriture. Elle a travaillé plusieurs années comme employée domestique et femme de ménage, comme toutes les femmes de sa famille. Deuxième d'une fratrie de neuf frères et sœurs, elle est la seule qui a fait des études supérieures. Dans les années 1970, elle déménage à Rio de Janeiro. Reçue au concours d'institutrice, elle exerce ce métier dans les années 1990. En 2011, elle soutient une thèse de doctorat en littérature comparée.

Conceição Evaristo, tout comme Carolina Maria de Jesus, a plus de quarante ans lorsqu'elle commence à publier ses fictions et ses mémoires. Elle a publié plus de sept livres dans plusieurs langues, dont plusieurs traduits en français. En 2018, elle était candidate à l'académie brésilienne de lettres, soutenue par une pétition qui a rassemblé plus de 22000 signatures $^{7}$. Elle innove avec un style qu'elle appelle elle-même " escrevivência » (écrivivance ou écrit-vie, un néologisme qui évoque l'écriture mélangée à l'expérience ou l'écriture de la vie) ${ }^{8}$. Comme Conceição Evaristo le définit : « il s'agit des fictions de la mémoire. Et, comme la mémoire oublie, on a besoin de l'invention ». (Evaristo C., 2017)

9 Dans cet article, j'envisage d'établir un parallèle entre le vécu de ces deux femmes noires écrivaines et de condition marginale au Brésil - même si elles deviennent «centrales » par la suite avec la reconnaissance de leur littérature -, en essayant de comprendre, à partir de leurs écrits, comment s'est construit une continuité entre ces deux projets dont la source était l'expérience de la misère et l'héritage de la condition d'esclaves au Brésil.L'article est structuré en trois parties : la première sur leur vécu dans leurs conditions de femmes; la deuxième sur l'expérience de la favela à leur époque et, enfin, la troisième sur les récits de mémoire de l'esclavage et sur la question raciale chez les deux écrivaines. Que disent-elles à travers leurs mémoires écrits sous la forme de journal ou de fiction inspirée de l'expérience ? Mon analyse porte notamment sur les ouvrages Quarto de Despejo (1960) [Le Dépotoir, 1962], de Carolina Maria de Jesus, et Becos da Memoria (2016) (traduit en français par Banzo, mémoires de la favela ${ }^{9}$ ), de Conceição Evaristo, et dans une moindre mesure sur les entretiens et témoignages des deux. Ces deux ouvrages ont été choisis car ce sont deux récits d'un temps - les années 1950, 1960 - sur le quotidien dans un espace commun - une favela d'une grande métropole, São Paulo (pour Carolina Maria de Jesus) et Belo Horizonte (pour Conceição Evaristo). Il s'agit également, dans les deux cas, des premiers ouvrages écrits par les deux autrices, même si, dans le cas de Conceição Evaristo, ce n'est pas son premier livre publié. J'ai choisi de travailler sur les versions en langue portugaise de ces ouvrages et de traduire moi-même en français les extraits cités ici ${ }^{10}$, puisque mon intérêt était de comprendre ces récits comme source de la condition des femmes afro-descendantes dans les favelas du Brésil au début du $\mathrm{xx}^{\mathrm{e}}$ siècle, et dans une moindre mesure de saisir aussi leur dimension littéraire, même si les deux ne sont pas séparées et si c'est justement la puissance esthétique qui donne force à leurs récits ${ }^{11}$. Mon propos était donc de dialoguer avec d'autres études sur la production littéraire des femmes afro- 
descendantes comprises dans leur double marginalité ${ }^{12}$, mais en privilégiant la littérature comme représentation ou comme source pour l'histoire ${ }^{13}$. Conceição Evaristo et Carolina Maria de Jesus sont agentes historiques de leur temps, insérées dans un réseau de sensibilités qui se renouvelle sans cesse selon les dynamiques sociales et politiques des différentes sociétés.

La question raciale qui apparaît dans ces récits est centrale pour comprendre le Brésil et la culture brésilienne. La mise en valeur du métissage et la façon dont se constitue "l'identité » brésilienne avec les apports de la culture portugaise et européenne, indigène et africaine a conduit soit à une invisibilité des différences raciales, soit à une vision d'égalité raciale (autrement dit, "démocratie raciale »). Ces interprétations, encore répandues à l'étranger, sont réfutées de façon catégorique par les intellectuels travaillant sur la question notamment depuis les années 1960 et par les militants brésiliens afro-descendants ${ }^{14}$. De nombreuses études démontrent l'existence d'un racisme structurel au Brésil, combiné aux ségrégations sociales ${ }^{15}$.

11 Le dernier pays du continent américain à avoir aboli l'esclavage (en 1888) est aussi celui qui a reçu le plus important contingent d'esclaves africains (presque 5 millions entre environ 1530 et 1850 , quand la traite transatlantique est interdite) ${ }^{16}$. L'esclavage, pendant presque quatre siècles, a été le principal élément structurant la vie économique et sociale du Brésil. De nos jours, de nouvelles approches revendiquent qu'on doit comprendre le Brésil, d'hier comme d'aujourd'hui, comme un grand pays esclavagiste et post-esclavagiste ${ }^{17}$. Étudier le post-abolitionnisme comme une problématique historique est aussi déterminant pour comprendre toutes les relations sociales et culturelles au Brésil, et s'interroger sur un autre prisme de l'« identité brésilienne » ou de la construction d'une compréhension de la construction de la nation avec et à partir de l'action d'autres agents historiques ${ }^{18}$.

12 Cet article souhaite aussi prendre en compte l'émergence de l'écriture des femmes noires dans le cadre des récentes revendications pour une autre visibilité des acteurs/ actrices marginaux. Depuis les années 2000 notamment, au Brésil, les politiques de « réparation » envers les afro-descendants voient le jour, comme notamment la loi des quotas universitaires et celle pour l'enseignement de l'histoire de l'Afrique, entre autres dispositifs de discrimination positive (actions affirmatives serait un terme plus juste) et de démocratisation de l'accès à la culture, aux savoirs, à la propriété (à travers les quilombos), à la mémoire ${ }^{19}$. Il s'agit aussi de prendre en compte le contexte de l'émergence de nouveaux intellectuels noirs et de nouvelles interprétations, avec un focus important sur le courant du féminisme noir, au Brésil et à l'étranger ${ }^{20}$. En France, nombre de ces études et fictions sont publiées grâce à l'action de la maison d'édition Anacaona, qui se positionne comme une véritable médiatrice de la question noire brésilienne dans la francophonie. Un récent ouvrage de la philosophe Djamila Ribeiro, revendique par ailleurs de prendre en considération la parole noire dans les productions culturelles et intellectuelles de l'occident, pour inverser les inégalités d'une pensée et de mode de fonctionnement colonisateurs ${ }^{21}$.

13 Si Conceição Evaristo est présente dans le paysage littéraire français contemporain elle a été reçue au salon du livre en 2015 et multiplie ses déplacements en France depuis -, Carolina Maria de Jesus n'est pas republiée en France, mais elle continue à être citée par d'autres auteur.e.s et dans plusieurs travaux en langue française. Au Brésil, on a de cesse de proposer sa relecture et de nouvelles études, des biographies, des maisons 
d'édition portant son nom, ou encore des ateliers et des concours littéraires inspirés de son action pionnière ${ }^{22}$.

\section{Être femme et favelada dans le Brésil des années 1950 et 1960 : le fil de l'écriture} C'est ainsi qu'elle écrit dans un passage, du 16 octobre 1958 : «Vous savez déjà que je vais chercher et porter de l'eau tous les jours. Maintenant, je vais changer le début de 
ma narration quotidienne.» (de Jesus, C. M., 1960, p. 125) Et c'est ainsi aussi que sa maison d'édition décide de terminer son livre, avec une seule ligne pour la journée du $1^{\text {er }}$ janvier 1960 : « Je me suis levée à cinq heures et je suis allée chercher de l'eau. » (de Jesus, C. M., 1960, p. 191)

Dans Becos da Memória, Conceição Evaristo écrit à la première personne, mais c'est une fiction. Son récit n'est pas directement un témoignage, mais, comme elle le dit dans la préface à la troisième édition du livre en portugais : "C'était mon premier essai de construire un texte fictionnel con-fondant écriture et vie, ou mieux, écriture et expérience » (Evaristo, C., 2017, p. 9). Dans ce livre, qui a été écrit en peu de temps mais qui n'a été publié qu'au bout de vingt ans (le livre est écrit en 1986 et publié pour la première fois en 2003), Conceição Evaristo développe son style de fiction parsemée de mémoires, ou de mémoire remplie par la fiction, comme elle le dit : avoir de «l'imagination pour remplir les vides des souvenirs transfigurés " (Evaristo, C., 2017, p. 11). Les souvenirs et la fiction portent sur le bidonville, aujourd'hui disparu, Pindura Saia où Conceição Evaristo a grandi, et qui a été détruit, dans la zone Sud de Belo Horizonte, dans les années 1950 et 1960. Sa construction narrative nous fournit des éléments sur un temps dans lequel Conceição Evaristo se rencontre avec son "eumenina » (Je/moi-fille). Donc le personnage de Maria-Nova - Marie-Jeune - fonctionne dans le livre comme une forme d'alter-ego, un personnage qui permet à Evaristo de s'introduire, dans une certaine mesure, dans sa fiction. Marie-Jeune est une petite fille intéressée par tout, gentille, cordiale et surtout très bonne élève, qui aime l'école, la lecture et l'écriture. Elle observe le monde de la favela, comme Conceição Evaristo le dit, c'est la petite Marie-Jeune qui va l'aider à mettre en fiction ses mémoires. A ce sujet, elle dit :

Quant aux similitudes de Marie-Jeune avec moi, à l'époque où j'étais jeune fille, je laisse l'énigme à ceux qui veulent bien la résoudre. J'insinue, seulement, que la littérature marquée par une écriture de la vie (escrevivência) peut con-fondre l'identité $\mathrm{du}$ personnage narrateur avec l'identité de l'autrice. Cette con-fusion ne me contraint pas.

(Evaristo, C., 2017, p. 12)

21 La fontaine collective est aussi très présente dans le récit d'Evaristo. Marie-Jeune était une observatrice privilégiée de ce qui se passait autour de la fontaine publique : «[Marie-Jeune] n'appréciait pas observer. Elle préférait la fontaine publique. Elle aimait bien voir l'agressivité des personnes les jours où l'eau était rare. Elle aimait entendre les histoires que les femmes racontaient tout bas » (Evaristo, C., 2017, p. 42).

Marie-Jeune aimait donc voir les événements et écouter les histoires autour de la fontaine publique, les histoires des femmes. La vie de la favela dépend des fontaines publiques à tel point que, pour faire partir la population définitivement lors du procès de démantèlement de la favela (desfavelamento), une des stratégies était de leur couper l'eau et l'accès aux fontaines, poursuit la narratrice dans Becos da Memória :

Rester sur place était devenu un enfer (...). Il y avait en plus la pénurie, le manque d'eau. Dans quelques habitations du quartier voisin, la nuit, le vigile donnait aux habitants de la favela quelques bidons d'eau. C'était un exercice fatigant. Nous marchions, souvent pendant presque une heure avec un bidon d'eau sur la tête et un autre dans les bras (...). Les trois dernières fontaines qui restaient coulaient très peu d'eau et quelques heures par jour seulement. Les blanchisseuses perdaient leurs clients.

(Evaristo, C., 2017, p. 156) 


\section{de ses trois enfants :}

Quel supplice de ramasser des papiers actuellement ! Je dois amener avec moi ma fille Vera Eunice. Elle a deux ans et n'aime pas rester à la maison. Je porte mon sac sur la tête et je la prends dans mes bras. Parfois, je me révolte. Ensuite, je maîtrise ma colère. Ce n'est pas de sa faute si elle est venue au monde. Ah, quelle est poignante la condition d'une femme seule sans un homme au foyer ! » (de Jesus, C. M., 1960, p. 22)

Les femmes sont très souvent victimes de violences, dans les deux récits sur les favelas brésiliennes des années 1950 et 1960. Carolina, malgré sa difficulté d'être seule parfois, se résigne à sa condition car au moins elle n'était pas victime de violences et des abus de la part de conjoints, comme elle le dit :

Elles, les autres femmes, doivent mendier et sont encore battues. On dirait du tambour. La nuit, quand elles appellent au secours, moi tranquillement j'écoute des valses viennoises. Pendant que leurs maris cassent leurs baraques, mes enfants et 
moi, nous dormons tranquillement. Je n'envie pas les femmes mariées qui mènent une vie d'esclaves indigènes.

(de Jesus, C. M., 1960, p. 16-17) un passage, elle raconte une discussion avec sa mère et son désir d'enfant de devenir un garçon, car même dans l'Histoire du Brésil, il n'y avait de place que pour les hommes :

Quand j'étais petite fille je rêvais de devenir un homme pour défendre le Brésil parce que je lisais l'Histoire du Brésil et j'apprenais des choses sur les guerres. Mais il n'y avait que des noms masculins cités comme défenseurs de la patrie. Je le disais donc à ma mère :

Pourquoi ne me transformez-vous pas en un homme?

Elle disait :

Si tu arrives à traverser un arc-en-ciel, tu deviendras un homme. »

(de Jesus, C. M., 1960, p. 53-54)

l'élément d'ouverture du premier roman de Conceição Evaristo, Poncia. Mais traverser l'arc-en-ciel était impossible pour le personnage Poncia, quand elle était encore petite fille et voulait devenir un garçon.

En ce qui concerne les métiers des femmes des favelas, dans les récits des deux écrivaines, on retrouve surtout trois possibilités : employées domestiques - métier exercé par les deux écrivaines -, prostituées ou mendiantes. Dans une certaine mesure, être chiffonnière était déjà un métier alternatif pour Carolina de Jesus avec lequel elle aura une relation ambivalente. Elle reconnaît sa pénibilité, mais parfois elle avoue aussi que ramasser des papiers ou du fer n'était pas en soi un problème pour elle. Le problème majeur était la misère et le fait d'habiter dans une favela :

Mon rêve était de bien m'habiller, avec des vêtements chers, d'habiter dans une maison confortable, mais cela n'est pas possible. Je ne suis pas mécontente avec le métier que j'exerce. Je me suis déshabituée à être sale. Cela fait déjà 8 ans que je ramasse du papier. Ce qui me rend mécontente, c'est d'habiter dans une favela.

(de Jesus, C. M., 1960, p. 22)

Dans un autre passage, Carolina de Jesus explore son quotidien de ramasseuse de papier de façon plus métaphorique : "On dirait que je suis venue au monde prédestinée à ramasser. La seule chose que je ne ramasse pas c'est le bonheur » (de Jesus, C. M., 1960, p. 53-54). Carolina de Jesus et Conceição Evaristo rêvent pourtant des lettres, de l'école, de devenir enseignantes ou, bien sûr, écrivaines.

Un recensement des habitants des favelas de Belo Horizonte de 1950, révèle, pour une population de 119799 personnes, que 86639 personnes, soit 72,31\%, étaient considérées inactives. Ces chiffres sont à prendre avec prudence, car dans la catégorie d'inactifs on inclut tous les mineurs, y compris les élèves scolarisés, les femmes au foyer et bien sûr les personnes exerçant des activités informelles. Parmi les personnes considérées actives $(27,69 \%)$, les femmes employées domestiques étaient représentées dans les différentes professions, comme les bonnes $(2,02 \%)$, les blanchisseuses $(1,87 \%)$, les employées domestiques $(1,47 \%)$, les cuisinières $(0,32 \%)$ et les couturières $(0,29 \%)^{25}$. 


\section{La favela dans les récits des femmes de la marge}

Une favela c'est le nom par lequel ont été connus les bidonvilles au Brésil. Tout d'abord à Rio de Janeiro, quand, après 1897, les soldats de retour de la campagne de Canudos (pour réprimer un peuplement dans le Nord-est du Brésil) ${ }^{26}$ ont fait une comparaison entre les habitations misérables qui se formaient dans la ville et la colline de Favela à Canudos. Ces occupations de bidonvilles sur les collines des grandes villes se sont multipliées partout au Brésil tout au long du $\mathrm{Xx}^{\mathrm{e}}$ siècle $^{27}$.

Dans les années 1950 et 1960, dates des récits de Carolina de Jesus et de Conceição Evaristo, les favelas étaient des endroits sans assainissement, en plein milieu urbain brésilien. Pour Carolina Maria de Jesus, la favela c'est le « dépotoir » ou l'arrière-cour de la ville : «Je classifie Sao Paulo comme ça : le siège du gouvernement, c'est le salon; la mairie, c'est la salle à manger ; et la ville c'est le jardin. La favela c'est l'arrière-cour où sont jetées les poubelles. » (de Jesus, C.M., 1960, p. 32.)

Conceição Evaristo, à travers le personnage de Marie-Jeune, la petite fille qui était une de rares habitantes de la favela qui fréquentait le collège, concrétise un autre parallèle entre la favela et la senzala, qui était le lieu d'habitation collective des esclaves dans les plantations au Brésil ${ }^{28}$. Le passage suivant est très révélateur du lien qu'il y a entre la condition de noir et de vie misérable dans une favela au Brésil au milieu du xxe siècle et être esclave dans une grande propriété :

Marie-Jeune divaguait dans une pensée lointaine et proche à la fois. Deux idées, deux réalités, deux images collées lui blessaient la poitrine. Senzala-favela. A cette époque-là, elle avait commencé ses études au collège, elle avait appris ce qu'était la casa-grande [Maison du maître]. (...) Elle voulait citer, comme exemple de casagrande, le quartier bourgeois voisin et, comme la senzala, la favela où elle vivait. Elle s'apprêtait à ouvrir la bouche, mais regarda sa classe et son institutrice. Chercha quelqu'un qui pourrait l'aider à soutenir cette idée et trouva la seule collègue noire qu'elle avait dans sa classe. Elle regarda la jeune fille, mais celle-ci avait la tête ailleurs comme si le sujet de l'esclavage n'avait rien à voir avec elle. [Marie-Jeune] sentit un certain malaise. Dans une classe de quarante-cinq élèves, il n'y avait que deux élèves noirs, et toutes les deux petites filles si éloignées l'une de l'autre. Elle se tut une fois de plus, mais cette pensée était toujours là. Senzalafavela, senzala-favela!

(Evaristo, C., 2017, p. 73) les conditions d'habitat peuvent être améliorées dans une certaine mesure dans les favelas au cours de ces années-là, avec l'arrivée de l'électricité, de l'eau courante et des conditions de vie un peu plus confortables, le trafic de drogue va changer complètement les réalités et les représentations de ces milieux marginaux. Nous pouvons émettre l'hypothèse selon laquelle la violence extrême et cette réalité du trafic remplace la famine comme élément structurant des nouveaux récits de la favela. Ces récits gardent, toutefois, les références à la misère et à la pauvretée ${ }^{29}$. 
Dieu veut bien m'aider, j'arriverai à déménager d'ici. J'espère que les hommes politiques élimineront les favelas ${ }^{30}$. »(de Jesus, C.M., 1960, p. 20)

41 Nous savons que Carolina quitte la favela en 1960, grâce au succès de ses livres, que la favela du Canindé disparaît à partir de 1961 et que tous ses habitants sont relogés dans une cité, dans un quartier éloigné du centre de Sao Paulo ${ }^{31}$. Pour Conceição Evaristo, la desfavelização est le grand élément structurant de son récit, qui narre les derniers moments pour les habitants dans la favela Pindura Saia. Le bidonville s'inscrit dans un processus de démantèlement qui commence à Belo Horizonte dans les années 1940, parfois avec la création des quartiers ouvriers et de "conjuntos habitacionais " (formes de cités HLM), en conformité avec la volonté des projets. Mais souvent, comme le dit le récit d'Evaristo, comme un moyen simplement d'expulser la population pauvre des zones de la ville destinées à devenir des quartiers aisés ${ }^{32}$. C'est le cas par ailleurs de la région de la favela Pindura Saia, qui donne lieu au quartier Anchieta à Belo Horizonte, une région aujourd'hui bourgeoise. Certes, la favela était un fléau, mais les habitants n'avaient pas d'autre endroit où aller, ce sont les mots de Conceição Evaristo, pour décrire le démantèlement de la favela :

Aux habitants, on offrait deux options: un peu de matériel de construction, des planches en bois et quelques briques pour qu'ils puissent construire leurs nouvelles baraques quelque part ailleurs, ou une indemnisation symbolique, un peu d'argent. Cette dernière option était la pire. Celui qui choisissait l'argent recevait un montant tellement modique qu'il le dépensait sur place. Et le pire était à venir, fini le délai de permanence, il ne restait ni argent, ni planches en bois, ni briques, il ne restait rien.

Tout le monde savait que la favela n'était pas le paradis, mais personne ne voulait la quitter. Le travail se trouvait à proximité, nos moyens de survie à tous aussi. Que ferions-nous dans ces endroits si éloignés où on nous obligeait à partir ? Il y avait des familles qui habitaient là-bas depuis des années, un demi-siècle peut-être, voire plus.

(Evaristo, C., 2017, p. 55)

Le récit de Conceição Evaristo révèle une réalité bien dure concernant les projets de desfavelização, en dépit de la propagande sur le sujet. Dans Becos da Memória, la petite Marie-Jeune regarde les travaux, la poussière, le «grand trou » provoqué par l'action des tracteurs, et le départ des différentes familles. Ils partaient loin du travail, loin de l'école des enfants. Certains étaient même obligés d'arrêter les études; parfois des familles entières devenaient mendiantes et sans-abri. C'était cela le destin de favelados " après la favela ».

\section{De la senzala à la favela : mémoire de l'esclavage et condition noire}

Dans un travail pionnier, les historiennes Hebe Mattos et Ana Lugão ont cherché à comprendre la mémoire de l'esclavage à travers le récit des premières générations des descendants d'esclaves dans le sud-est du Brésil, et plus précisément dans la région de plantation de café du Vale de Paraíba ${ }^{33}$. Les entretiens ont été faits dans les années 1980 et 1990, auprès des descendants qui étaient restés dans le milieu rural (pas ceux qui avaient migré dans les villes et avaient construit et vécu dans les taudis et les favelas).

Dans des conditions toujours dures et parfois misérables, les descendants d'esclaves révèlent toutefois deux sens attribués à la liberté dès la proclamation de l'abolition de 
l'esclavage : le fait de disposer de sa mobilité et de pouvoir déménager éventuellement, et le fait aussi de n'être plus assujettis aux châtiments corporels. Conceição Evaristo, dans Becos da Memória, nous livre plusieurs éléments sur ces questions de la liberté et du passé esclavagiste. Un des personnages, Luis, était un ancien esclave ou descendant d'esclaves dont la famille n'avait pas été mise au courant de l'abolition et de leurs droits :

Luis fut un garçon intelligent, toujours curieux des choses et des causes. C'était un rebelle, il détestait les maîtres. Quand on a vendu sa sœur, parce qu'elle avait tiré son maître par la chemise, il vomissait sa haine et promettait de se venger et de mettre le feu à la maison des maîtres. (...) Le lendemain, Luis disparut (...). Un jour, comme si de rien n'était, le garçon revint, c'était déjà un jeune homme (...) - Papa, sortons d'ici, nous n'avons pas besoin de prévenir le maître. Dans mes pérégrinations, j'ai découvert des choses... Cela fait longtemps que les Blancs ne possèdent plus les Noirs. »

(Evaristo, C., 2017, p. 34)

Carolina de Jesus quant à elle mentionne la conscience de cet héritage quand elle célèbre le 13 mai, date officielle de l'abolition de l'esclavage au Brésil, tout en montrant l'ambiguïté de son statut de descendante d'esclave, libre, mais tellement misérable. L'égalité citoyenne n'était qu'une promesse :

Le 13 mai [1958]. Aujourd'hui, le jour s'est levé sous la pluie. C'est un jour sympathique pour moi. C'est le jour de la libération des esclaves. (...) Dans les prisons, les Noirs étaient des cobayes. Mais maintenant les Blancs sont plus cultivés et ils ne nous traitent plus avec mépris. Que Dieu puisse illuminer les Blancs pour que les Noirs puissent être heureux.

(...) Il était 9 heures du soir quand nous avons pu manger. Et de cette façon, le 13 mai 1958 je luttais contre l'esclavage actuel - la faim !

(Evaristo, C., 2017, p. 30)

Cette pénurie de moyens pour lutter pour l'égalité, pour la dignité, est représentée dans l'écriture de Carolina Maria de Jesus par un élément crucial, le manque de chaussures pour elle et pour ses enfants. L'absence des chaussures est un important stigmate de l'esclavage au Brésil (Chalhoub, S., 1990). J'ai comptabilisé au moins six passages importants dans lesquels Carolina de Jesus nous parle de sa difficulté à se procurer des chaussures. Le sujet est abordé dès l'ouverture de son livre :

Le 15 juin [1955]. C'est l'anniversaire de ma fille Vera Eunice. J'avais l'intention de lui acheter une paire de chaussures. Mais le coût des denrées alimentaires nous empêche de réaliser nos désirs. J'ai trouvé une paire de chaussures dans la poubelle, je les ai lavées et je les ai réparées afin qu'elle puisse les mettre.

(De Jesus, C.M., 1960, p. 11)

Ou encore, quelques années plus tard, le 28 mai 1958 :

La journée a débuté sous la pluie. J'ai seulement treize cruzeiros car j'en ai prêté cinq à Leila pour qu'elle puisse aller chercher sa fille à l'hôpital. Je suis désorientée, sans savoir ce par quoi je peux commencer. Je veux écrire, je veux travailler, je veux laver le linge. J'ai froid. Et je n'ai pas de chaussures à me mettre. Les chaussures de mes enfants sont trouées.

(De Jesus, C.M., 1960, p. 45)

La question de la dignité des vêtements et du manque de chaussures apparait chez Conceição Evaristo dans le personnage de Ditinha, qui devient une employée domestique d'une famille aisée. Elle observe les habits et les chaussures de sa patronne et s'interroge sur elle-même, en se regardant dans la glace : «Elle se regarda dans la glace et se sentit tellement moche, encore plus moche que d'habitude. "Et si j'avais des robes et des chaussures, et si je savais me coiffer ?" (Ditinha détestait ses cheveux).» 
(Evaristo, C., 2017, p. 34) d'interrogations profondes sur la condition des Noirs au Brésil : la condition de l'égalité ou pas, la question du pouvoir économique, la supériorité esthétique et même les privilèges dans toutes les relations humaines. Comme le démontre Djamila Ribeiro, entre autres auteurs, la race et la classe ne sont pas dissociées au Brésil, on ne peut pas les séparer (Ribeiro, D., 2019). Être pauvre, être favelado et être noir sont des conditions associées. Et une barrière difficile à surmonter, même si les personnages ou autrices sont fières d'être des femmes noires. Ainsi, un passage de Carolina de Jesus est extrêmement révélateur de ces éléments, le 16 juin 1958 :

J'écrivais des pièces et je les montrais aux directeurs des cirques. Ils me répondaient : «C'est dommage que vous soyez noire."

Ils oubliaient cependant que j'adore ma peau noire, et mes cheveux rustiques. (...) Si la réincarnation existait, je voudrais toujours revenir au monde comme noire.

(De Jesus, C.M., 1960, p. 64)

\section{Carolina Maria de Jesus écrit dans un très fort passage :}

Le 28 mai [1959]. La vie est comme un livre. C'est seulement après l'avoir lu en entier que nous savons ce qui nous attend pour la fin. Et c'est seulement quand nous sommes à la fin de la vie, que nous savons comment notre vie s'est passée. La mienne, jusqu'ici, est noire. Noire est ma peau. Noir c'est l'endroit où j'habite.

(De Jesus, C.M., 1960, p. 167)

Conceição Evaristo expose la question raciale à partir de ses personnages, des habitants de la favela. Ces personnages expriment aussi cette dualité : être noir est une expérience vécue comme une fierté, mais aussi une condition qui signifie être victime d'un racisme virulent des blancs qui gardaient le pouvoir économique et social. C'est ainsi qu'elle raconte l'instant même où un nouveau couple se rencontre, Dora et Negro Alírio (Noir Alírio) :

Elle a dit qu'elle s'appelait Doria (...). Il a dit qu'il s'appelait Noir Alírio. Cela lui a fait plaisir d'entendre le mot noir prononcé par un Noir, puisqu'elle n'entendait ce terme que dans la bouche des Blancs, et toujours pour insulter : Noir bâtard ; Noir fils de pute ; Noir bagarreur et tant d'autres défauts.

(Evaristo, C., 2017, p. 95)

51 Les relations raciales et sociales étaient aussi définies par les intérêts et les enjeux politiques. Dans un long passage à la première personne, Conceição Evaristo parle des candidats aux élections qui venaient à la favela, avec beaucoup de scepticisme et d'indignation :

En période d'élection, apparaissaient sur place des candidats qui juraient qu'ils allaient faire quelque chose pour nous. (...) Leurs propagandes, vieux journaux, tracts, après être lus mot à mot, quand ils étaient lus, accomplissaient une autre fonction : ils servaient à nettoyer nos fesses. Les visages et les yeux de tous ces candidats que nous n'avions jamais vus précédemment et que nous ne verrions plus jamais, surtout s'ils étaient victorieux dans les urnes, nous persécutaient (...). Les femmes et les enfants de la favela s'amusaient à voter pour les candidats ayant les plus beaux visages. Un jour, un candidat Noir est venu. (...) Il paraissait être pauvre comme nous. Lors du concours de beauté, il a obtenu peu de voix.

Ceux qui n'étaient pas élus revenaient souvent lors d'autres occasions avec les mêmes demandes et les mêmes promesses. (...) Ils demandaient ce que les autres faisaient pour nous. Rien! Eux-mêmes répondaient. Ils ne voulaient même pas entendre nos voix. (...) Parfois ils gagnaient ; et quand cela se produisait notre situation restait pareille, nous étions ceux qui ne gagnaient jamais. 
(Evaristo, C., 2017, p. 118) étaient exclus de la politique. Les candidats et hommes politiques étaient les autres, des personnes extérieures à la favela, des inconnus, et des imposteurs. Les solutions pour la favela ne venaient pas des urnes. Dans une telle situation d'exclusion, les habitants des favelas ne se sentaient pas responsables de la misère de leur vie ni de celle de leur monde, comme Carolina de Jesus le reconnaît dans cet intéressant passage : « Le monde est tel que les Blancs le veulent. Je ne suis pas blanche, je n'ai rien à voir avec ça.» (de Jesus, C.M., 1960, p. 70)

\section{Conclusion : Écrire pour témoigner et pour survivre}

Si Carolina Maria de Jesus produit un journal, Conceição Evaristo écrit une «fiction mémorielle ». Mais le désir d'écrire est un élément qui unit ces deux femmes. L'acte de l'énonciation est aussi fort que le contenu de leurs récits, non seulement parce que ces deux femmes osent écrire, mais aussi parce qu'elles osent créer et s'inscrire dans une tradition littéraire, dans un canon littéraire d'autres femmes afro-descendantes, ce qui est d'autant plus délibéré pour Conceição Evaristo à travers ses personnages (comme Ditinha, par exemple). Prendre la plume pour dénoncer, pour résister, pour créer, pour revendiquer une place dans la société de la culture car l'écriture est aussi un droit ${ }^{34}$. L'écriture était également un refuge, comme le dit Carolina de Jesus dans ce passage de son journal : "Quand je suis énervée, je n'aime pas discuter. Je préfère écrire. J'écris tous les jours. Je m'assieds dans l'arrière-cour et j'écris. » (Cândido, A., 2011, p. 22)

Conceição Evaristo nous parle aussi de son goût pour la lecture, de son intérêt pour les livres depuis l'enfance, à travers le personnage de Marie Jeune : "petite encore, elle épluchait pendant des heures et des heures les revues et journaux offerts par sa mère et sa tante. » (Evaristo, C., 2017, p. 99)

Un désir qui était transmis par ses ancêtres, comme sa mère qui « a appris seule à lire en repérant soigneusement les lettres (...). C'était peut-être pour cette raison qu'elle désirait tant que ses enfants apprennent à lire et y mettait toute son énergie. " (Evaristo, C., 2017, p. 64)

Devenir enseignante est aussi un des chemins empruntés par Conceição Evaristo, qui devient plus tard docteure en littérature, tout comme la fille de Carolina de Jesus, Vera Eunice, qui est devenue institutrice.

Toutefois, comprendre la profondeur et l'importance de l'œuvre de ces deux écrivaines nous invite aussi à réfléchir sur la création d'un nouveau projet littéraire et d'un nouveau style littéraire, comme Conceição Evaristo le fait remarquer quand elle s'est exprimée récemment sur le travail de Carolina Maria de Jesus. Carolina de Jesus est précurseur d'une tradition et d'une création de style littéraire, quand elle parle, entre autres, de la faim. Sa faim, nous rappelle Conceição Evaristo, n'est pas uniquement une faim physique, c'est une faim politique et symbolique, un désir de transformation sociale par la littérature qui dépasse sa réalité ${ }^{35}$. Ce sont donc deux conditions de vie marginales - Carolina Maria de Jesus et Conceição Evaristo - qui sont le moteur (sur la forme comme sur le fond), d'une création esthétique et littéraire nouvelle et transgressive.

Annales, Histoire, sciences sociales, 65a . Année, no. 2, mars-avril 2010

IdeAs, 16 | 2020 
Cândido, Antônio, $\mathrm{O}$ direito à literatura. In: Vários escritos, Rio de Janeiro, Ouro Sobre Azul, 2011, p. 171-183.

Castro, Josué, Geografia da fome : o dilema brasileiro, Rio de Janeiro, Antares, 1984.

Cooper, Frederick, Holt, Thomas C., Scott, Rebecca, Beyond Slavery, explorations of race, labor and citizenship in postemancipation societies, University of North Carolina Press, 2000.

\section{BIBLIOGRAPHIE}

Evaristo, Conceição, L'histoire de Poncia, Paris, Anacaona, 2015.

Evaristo, Conceição, Becos da memória, Rio de Janeiro, Pallas, 2017.

De Jesus, Carolina Maria, Quarto de despejo, São Paulo, Ática, 2014. Publié en français sous le titre : de Jesus, Carolina Maria, Le Dépotoir, Paris, Stock, 1962.

De Jesus, Carolina Maria, Le journal de Bitita, Paris, Métaillé, 1982.

Almeida, Silvio Luiz de, O que é racismo estrutural, Belo Horizonte, Letramento, 2018.

Capanema P. De Almeida, Silvia, Flechet, Anaïs (orgs.), De la démocratie raciale au multiculturalisme : Brésil, Amériques, Europe, Bruxelles, Peter Lang, 2009.

Chartier, Roger. «Le monde comme représentation ». In : Annales, Histoire et Sciences Sociales : un tournant critique, $n^{\circ} 44-6,1989$, p. 1505-1520.

Cunha, Euclides da, Hautes Terres. La guerre de Canudos, Paris, Métaillé, 2012.

Hebrard, Jean (org.), Brésil, quatre siècles d'esclavage, Paris, Karthala, 2012.

Hook, Bell et al., Black feminism : anthologie du féminisme africain-américain, Paris, l'Harmattan, 2008.

Farias, Tom, Carolina: uma biografia, São Paulo, Malê, 2018.

Lyon-Caen, Judith, La griffe du temps. Ce que l'histoire peut dire de la littérature, Paris, Gallimard, 2019.

Mattos, Hebe, Les couleurs du silence, Esclavage et liberté dans le Brésil du XIX $\mathrm{X}^{\mathrm{e}}$ siècle, Paris, Karthala, 2019

Mattos, Hebe, Rios, Ana Lugao, Memorias do Cativeiro: família, trabalho e cidadania no proabolição, Rio de Janeiro, Civilização Brasileira, 2005.

Mbembe, Achille, Critique de la raison nègre, Paris, La Découverte, 2013.

Ribeiro, Djamila, La place de la parole noire, Paris, Anacaona, 2019.

Ribeiro, Djamila, Petit manuel antiraciste et féministe, Paris, Anacaona, 2020.

Schwarcz, Lilia M. et Gomes, Flavio (org.), Dicionário da escravidão e liberdade, São Paulo, Cia das Letras, 2018.

Salgueiro, Maria Aparecida Andrade, « Desafiando a dupla exclusão - a ficção de autoras afrodescendentes », Saúde, Sexo e Educação, Rio de Janeiro, vol. ano XIV, n 37, p. 7-13, 2005. 
Sousa, Germana H. Pereira de, “A tradução francesa da linguagem compósita de Carolina Maria de Jesus", Cadernos de Tradução, Florianópolis, vol. 2, n² 28, p. 121, 2011.

Souza, Jessé, A elite do atraso: da escravidão à lava jato, Rio de Janeiro, Leya, 2017.

\section{NOTES}

1. Voir l'analyse du journaliste Audálio Dantas dans la préface de: Jesus, Carolina Maria de, Quarto de despejo, São Paulo, Atica, 2014, p. 7.

2. Jesus, Carolina Maria de, Quarto de despejo, op. cit. Edition en français : De Jesus, Carolina Maria de, Le Dépotoir, Paris, Stock, 1962.

3. Le reportage avec les textes de Carolina Maria de Jesus publié dans la revue $O$ Cruzeiro a été récemment republié sur une revue en ligne : https://medium.com/revista-figas/retrato-dafavela-no-diário-de-carolina-8931572045ae (consulté le 20/03/2020).

4. Sur la vie de Carolina Maria de Jesus, voir : Farias, Tom, Carolina: uma biografia, São Paulo, Malê, 2018.

5. Jesus, Carolina Maria de, Le journal de Bitita, Paris, Métaillé, 1982. Sur ce journal, voir l'essai de Germana de Sousa présenté lors du colloque « Mémoire des Amériques », en juin 2007, et non publié : $\quad$ https://repositorio.unb.br/bitstream/10482/9193/1/ CAPITULO_LetrangeJournalCarolina.pdf (consulté le 20/03/2020).

6. Sur la vie de Conceição Evaristo, voir l'avant-propos dans : Evaristo, Conceição, L'histoire de Poncia, op. cit.

7. Voir : https://www.change.org/p/queremos-conceiçao-evaristo-na-academia-brasileira-deletras?use_react=false. (Consulté le 01/06/2020).

8. Paula Anacaona propose de traduire escrivivência par "écrit-vie " (Evaristo, Conceição, L'histoire de Poncia, op. cit), alors que Izabella Borges le définit comme «écriture de la vie, du vécu ", dans le dictionnaire des créatrices: https://www.dictionnaire-creatrices.com/ficheconceicao-evaristo?q=Concei\%C3\%A7\%C3\%A3o+EVARISTO (consulté le 10/08/2020).

9. Evaristo, Conceição, Banzo, mémoires de la favela, Paris, Anacaona, 2016.

10. Sur la critique de la traduction française de Quarto de Despejo, voir: Sousa, Germana H. Pereira de, A tradução francesa da linguagem compósita de Carolina Maria de Jesus, Cadernos de Tradução, Florianópolis, vol. 2, n 28, 2011, p. 121.

11. Sur les relations entre littérature et histoire et notamment sur l'usage de la littérature comme source pour l'histoire, voir : «Savoirs de la littérature », Annales, Histoire, sciences sociales,

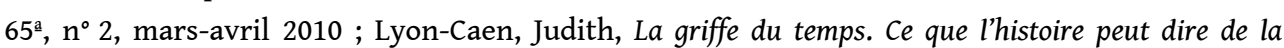
littérature, Paris, Gallimard, 2019.

12. Voir, entre autres : Salgueiro, Maria Aparecida Andrade. «Desafiando a dupla exclusão - a ficção de autoras afro-descendentes ", Saúde, Sexo e Educação. Rio de Janeiro, vol. ano XIV, n 37, p. 7-13, 2005.

13. Sur le concept de représentation, voir : Chartier, Roger. "Le monde comme représentation ». In : Annales, Histoire et Sciences Sociales : un tournant critique, $n^{\circ} 44-6,1989$, p. 1505-1520.

14. Sur cette question de la "démocratie raciale» brésilienne à son refus dans les analyses contemporaines, voir, parmi d'autres : Capanema P. De Almeida, Silvia ; Flechet, Anaïs (orgs.), De la démocratie raciale au multiculturalisme : Brésil, Amériques, Europe, Bruxelles, Peter Lang, 2009.

15. Il est important de citer ici, parmi plusieurs autres travaux, la mise à jour sur le sujet faite dans : Almeida, Silvio Luiz de, o que é racismo estrutural, Belo Horizonte, Letramento, 2018.

16. Un ouvrage récent, non traduit en français, reprend une bonne partie des analyses contemporaines sur le sujet : Schwarcz, Lilia M. \& Gomes, Flavio (org.), Dicionário da escravidao e liberdade, Sao Paulo, Cia das Letras, 2018. En langue française, voir aussi : Hebrard, Jean (org.), 
Brésil, quatre siècles d'esclavage, Paris, Karthala, 2012. Enfin, le récent livre d'Hebe Mattos qui met en évidence les pratiques de nomination et reconnaissance, ainsi que l'invisibilité de la couleur de la peau, pendant les dernières années de l'esclavagisme : Mattos, Hebe, Les couleurs du silence, Esclavage et liberté dans le Brésil du XIxe siècle, Paris, Karthala, 2019.

17. Souza, Jessé, A elite do atraso: da escravidão à lava jato, Rio de Janeiro, Leya, 2017.

18. Sur la définition de période post-abolitionniste voir, entre autres : Cooper, Frederick, Holt, Thomas C., Scott, Rebecca, Beyond Slavery, explorations of race, labor and citizenship in postemancipation societies, University of North Carolina Press, 2000.

19. Voir aussi : CAPANEMA P. DE ALMEIDA, S. ; FLECHET, A., De la démocratie raciale..., op. cit.

20. Sur cette question, voir : Hooks, Bel et alii, Black feminism : anthologie du féminisme africainaméricain, Paris, l'Harmattan, 2008. Voir aussi : Ribeiro, Djamila, La place de la parole noire, Paris, Anacaona, 2019 ; Ribeiro, Djamila, Petit manuel antiraciste et féministe, Paris, Anacaona, 2020.

21. Ribeiro, Djamila, La place de la parole noire, op. cit. De façon comparative et concernant la critique d'une soumission à la pensée européenne et occidentale, ce changement de perspective se trouve aussi au centre du travail de: Mbembe, Achille, Critique de la raison nègre, Paris, La Découverte, 2013.

22. L'édition numérique de la FLUP (fête littéraire des périphéries) de 2020 est dédié à Carolina Maria de Jesus et aux soixante ans de publication de Quarto de Despejo. Voir : https:// oglobo.globo.com/cultura/nos-60-anos-de-quarto-de-despejo-autoras-da-flup-escrevemcarolina-de-jesus-24421873 (Consulté le 03/06/2020).

23. Sur la faim au Brésil, voir le classique de Josué de Castro: Castro, Josué Geografia da fome: o dilema brasileiro, Rio de Janeiro, Antares, 1984.

24. https://www.bol.uol.com.br/noticias/bbc/2019/03/02/maria-lata-dagua-a-passista-quesambava-com-20-litros-de-agua-na-cabeca.htm. (Consulté le 02/06/2020).

25. Oliveira, Samuel Silva Rodrigues de, "Trabalhadores favelados": identificação das favelas e movimentos sociais no Rio de Janeiro e Belo Horizonte, Thèse de doctorat en histoire, Fundação Getúlio Vargas, Rio de Janeiro, setembro 2014, p. 131.

26. Cunha, Euclides da, Hautes Terres. La guerre de Canudos, Paris, Métaillé 2012.

27. Sur les favelas brésiliennes dans le temps, voir l'excellent ouvrage de Licia Valladares : Valladares, Licia, La favela d'un siècle à l'autre, Paris, MSH, 2006.

28. Cette opposition entre la maison des maîtres (Casa-Grande) et celle des esclaves (Senzala) comme structurante de la société brésilienne, même si avec des "passages» possibles, est représentée dans le classique de Gilberto Freyre (y compris dans son titre original, Casa-grande e senzala) : FREYRE, Gilberto, Maîtres et Esclaves, Paris, Gallimard, coll. Tel, Paris 1978.

29. De nombreuses fictions, séries et films sur la violence dans les favelas liée au trafic de drogues existent à partir des années 1970 et 1980, comme, par exemple : Lins, Paulo, Cidade de Deus, São Paulo, Companhia das Letras, 1997.

30. De Jesus, Carolina Maria de, Quarto de despejo, op. cit., p.20.

31. Sur ces processus, voir : Valladares, Licia, op. Cit.

32. Oliveira, Samuel Silva Rodrigues de, op. cit.

33. Le projet de recherche est publié sur un site internet et a donné origine à une série de documentaires sur la mémoire des descendants et les expressions culturelles des régions des Quilombos (terres de communautés d'esclaves marrons aujourd'hui appartenant aux descendants d'esclaves). Voir: http://www.labhoi.uff.br/narrativas/home. Voir aussi le livre: Mattos, Hebe, Rios, Ana Lugão, Memórias do Cativeiro : familia, trabalho e cidadania no pro-abolição, Rio de Janeiro, Civilização Brasileira, 2005.

34. Je pense ici à un prolongement du «droit à la littérature" pensé par Antônio Cândido : Cândido, Antônio, O direito à literatura. In : Vários escritos, Rio de Janeiro, Ouro Sobre Azul, 2011, p. 171-183. 
35. Débat entre Conceição Evaristo e Vera Eunice, fille de Carolina Maria de Jesus, pour l'ouverture de la FLUP numérique en hommage à Carolina, le 16 mai 2020, disponible sur : https://www.youtube.com/watch?v=V7c5IWcB_dQ (consulté le 20/05/2020).

\section{RÉSUMÉS}

L'article analyse, de façon croisée et en proposant de comparer le parcours et l'écriture, comme témoignage, de deux écrivaines brésiliennes originaires des favelas : Carolina Maria de Jesus (1914-1977) et Conceição Evaristo (1946-...).

Leur écriture est ainsi comprise en tant que source et témoignage pour les sciences humaines et pour l'histoire, dans une étude qui privilégie leur trajectoire en tant qu'actrices sociales et femmes noires des favelas devenues écrivaines dans des contextes différents. Bien que pratiquant un style différent - Carolina de Jesus a produit un journal, alors que Conceição Evaristo a rédigé une œuvre de fiction (un roman contemporain avec une importante dimension autobiographique) -, les travaux et l'histoire de la vie des deux écrivaines peuvent être mis en perspective. Leur écriture nous livre d'importants éléments pour comprendre le quotidien des afro-descendants dans les favelas brésiliennes, en particulier des femmes, leur condition marginale, vue de l'intérieur. La richesse des éléments et l'originalité de ces deux écritures «mémorielles » s'expliquent aussi par le fait que ces deux femmes sont observatrices et actrices d'une réalité marginale de leur temps, pour produire une littérature engagée et de transformation sociale.

The essay analyzes and compares the trajectories and the works of two Brazilian female writers born in the favelas.

The article does not purport to offer a literary analysis of the works; rather, it aims at examining them as sources that can be used in social sciences and history. The essay will analyze the trajectories of these two black female social players who became writers in different contexts. While the genre of their works differs - Carolina de Jesus wrote a diary, and Conceição Evaristo wrote fiction (a contemporary novel with an important autobiographical dimension) - it is interesting to put the two women and their life stories in perspective. Their work allows us to gain considerable insight into the experience of women of African descent, into their lives in the Brazilian favelas and their marginal situation as seen from within.

The wealth and originality of these works can also be accounted for by the fact these women are both witnesses of and players in a life on the margins; this is what they convey in type of literature that has a message, that tries to address social problems and transform society.

$\mathrm{O}$ artigo analisa, de forma cruzada e propondo comparar o percurso e a escrita como testemunho de duas escritoras brasileiras oriundas da favela: Carolina Maria de Jesus (1914-1977) e Conceição Evaristo (1946-...).

Através dos escritos dessas duas escritoras tomados como fonte e testemunho para as ciências humanas e para a história, propõe-se um estudo que privilegia suas trajetórias como agentes sociais e mulheres negras da favela que se tornaram escritoras em contextos diferentes. Ainda que praticando estilos diferentes - Carolina de Jesus produz um diário e Conceição Evaristo uma obra de ficção contemporânea com grande inspiração autobiográfica -, os trabalhos e as histórias de vida das duas escritoras podem ser colocados em perspectiva. Ambas escritas nos fornecem 
elementos importantes para se compreender o cotidiano nas favelas brasileiras, sua condição marginal, visto do interior. A riqueza dos elementos e a originalidade das duas escritas "memoriais" se explicam também pelo fato de que as duas mulheres são observadoras e agentes de uma realidade marginal de suas épocas, realidade esta que tentam representar através de uma forma literária engajada e que reivindica a necessidade de transformação social.

\section{INDEX}

Mots-clés : Favela, écriture, femmes noires, héritage de l'esclavage

Palavras-chave : Favela, escrita, mulheres negras, herança escravista

Keywords : Favela, writing, black women, slavery legacy

\section{AUTEUR}

\section{SILVIA CAPANEMA}

Docteure en Histoire par l'EHESS, Paris, Maîtresse de conférences à l'Université Paris 13 Sorbonne Paris NordChercheuse à Pléiade, Campus Condorcet 